\title{
Selections from international journals
}

\author{
Nahla M. Heshmat \\ Professor of Pediatrics, Ain Shams University
}

\section{J Allergy Clin Immunol. 2019;143(2):651-661.}

\section{Multicenter, randomized, double-blind, placebo-controlled clinical trial of vital wheat gluten oral immunotherapy.}

\author{
Nowak-Węgrzyn A, Wood RA, Nadeau KC, Pongracic JA, Henning AK, Lindblad RW, Beyer K, Sampson
} HA.

BACKGROUND: Wheat is a common food allergen that can cause anaphylaxis. OBJECTIVE: We sought to determine the efficacy and safety of vital wheat gluten (VWG) oral immunotherapy (OIT). METHODS: After baseline double-blind, placebo-controlled food challenge (DBPCFC), 46 patients with wheat allergy (median age, 8.7 years; range, 4.2-22.3 years) were randomized 1:1 to low-dose VWG OIT or placebo, with biweekly escalation to $1445 \mathrm{mg}$ of wheat protein (WP). After a year $1 \mathrm{DBPCFC}$, active subjects continued low-dose VWG OIT for another year and underwent a year 2 DBPCFC and, if passed, a subsequent off-therapy DBPCFC. Placebo-treated subjects crossed over to high-dose VWG OIT (maximum, $2748 \mathrm{mg}$ of WP). RESULTS: The median baseline successfully consumed dose (SCD) was $43 \mathrm{mg}$ of WP in both groups. At year 1, 12 (52.2\%) of 23 low-dose VWG OIT-treated and $0(0 \%)$ of 23 placebo-treated subjects achieved the primary end point of an SCD of $4443 \mathrm{mg}$ of WP or greater ( $<$.0001); median SCDs were 4443 and $143 \mathrm{mg}$, respectively. At year 2, $7(30.4 \%)$ of 23 lowdose VWG OIT-treated subjects were desensitized to an SCD of $7443 \mathrm{mg}$ of WP; 3 (13\%) achieved sustained unresponsiveness 8 to 10 weeks off therapy. Among placebo-treated subjects who crossed over to high-dose VWG OIT, $12(57.1 \%)$ of 21 were desensitized after 1 year (median SCD, $7443 \mathrm{mg}$ of WP; nonsignificant vs low-dose VWG OIT). At year 1, skin prick test responses and wheat- and omega-5 gliadin-specific IgE levels did not differ between groups; the low-dose VWG OIT median specific IgG4 level was greater than placebo (wheat, $\mathrm{P}=.0005$; omega-5 gliadin, $\mathrm{P}=.0001)$. Year $1 \mathrm{SCDs}$ correlated with wheat-specific $($ rho $=0.55, \mathrm{P}=.0003)$ and omega-5 gliadin-specific (rho $=0.51, \mathrm{P}=.001$ ) IgG4levels in all subjects. Among 7822 low-dose VWG OIT doses in year $1,15.4 \%$ were associated with adverse reactions: $0.04 \%$ were severe, and $0.08 \%$ subjects received epinephrine. Among 7921 placebo doses, $5.8 \%$ were associated with adverse reactions; none were severe. CONCLUSIONS: Low- and high-dose VWG OIT induced desensitization in about one half of the subjects after 1 year of treatment. Two years of low-dose VWG OIT resulted in 30\% desensitization, and 13\% had sustained unresponsiveness.

\section{J Allergy Clin Immunol. 2018;141(2):529-538.}

\section{Results from the 5-year SQ grass sublingual immunotherapy tablet asthma prevention (GAP) trial in children with grass pollen allergy.}

\author{
Valovirta E, Petersen TH, Piotrowska T, Laursen MK, Andersen JS, Sørensen HF, Klink R; GAP \\ investigators.
}

BACKGROUND: Allergy immunotherapy targets the immunological cause of allergic rhinoconjunctivitis and allergic asthma and has the potential to alter the natural course of allergic disease. OBJECTIVE: The primary objective was to investigate the effect of the SQ grass sublingual immunotherapy tablet compared with placebo on the risk of developing asthma. METHODS: A total of 812 children (5-12 years), with a clinically relevant history of grass pollen allergic rhinoconjunctivitis and no medical history or signs of asthma, were included in the randomized, double-blind, placebo-controlled trial, comprising 3 years of treatment and 2 years of follow-up. RESULTS: There was no difference in time to onset of asthma, defined by prespecified asthma criteria relying on documented reversible impairment of lung function (primary endpoint). Treatment with the SQ grass sublingual immunotherapy tablet significantly reduced the risk of experiencing asthma symptoms or using asthma medication at the end of trial (odds ratio $=0.66, \mathrm{P}<.036$ ), during the 2 -year posttreatment follow-up, and during the entire 5year trial period. Also, grass allergic rhinoconjunctivitis symptoms were $22 \%$ to $30 \%$ reduced $(\mathrm{P}<.005$ for all 5 years). At the end of the trial, the use of allergic rhinoconjunctivitis pharmacotherapy was significantly less (27\% relative difference to placebo, $\mathrm{P}<.001$ ). Total IgE, grass pollen-specific IgE, and skin prick test reactivity to grass 
pollen were all reduced compared to placebo. CONCLUSIONS: Treatment with the SQ grass sublingual immunotherapy tablet reduced the risk of experiencing asthma symptoms and using asthma medication, and had a positive, long-term clinical effect on rhinoconjunctivitis symptoms and medication use but did not show an effect on the time to onset of asthma.

\title{
J Allergy Clin Immunol Pract. 2019;7(2):444-450.
}

\section{Food Protein-Induced Enterocolitis Syndrome Food Challenges: Experience from a Large Referral Center.}

\author{
Wang KY, Lee J, Cianferoni A, Ruffner MA, Dean A, Molleston JM, Pawlowski NA, Heimall J, Saltzman \\ RW, Ram GS, Fiedler J, Gober LM, Spergel JM, Brown-Whitehorn TF.
}

BACKGROUND: Food protein-induced enterocolitis syndrome (FPIES) is a non-IgE-mediated food allergy that is diagnosed based on clinical findings but can be confirmed with oral food challenge (OFC). OFC is more often performed to assess the development of tolerance. Most studies describing OFCs in FPIES are limited in size. OBJECTIVE: We sought to describe our experience with OFCs using our FPIES protocol. Patients were given one-third of serving size with a 4-hour observation period, followed by home titration to full dose. METHODS: We conducted a retrospective chart review of patients who underwent OFC via the FPIES protocol from 2014 to 2017. Data regarding the history of reaction, age at the time of challenge, and reactions during challenge or with home introduction were collected. RESULTS: A total of 169 OFCs were completed under the FPIES protocol, in 119 patients to 19 different foods. Thirty challenges (18\%) were positive, with 17 challenges (10\%) during initial challenge and 13 (7.7\%) during home dosing. Most reactions during initial challenge required intravenous fluids (IVF), but hypotension was uncommon. One hundred thirty-nine $(82 \%)$ OFCs were negative with home introduction, indicating tolerance to the challenged foods. The mean age of passing a challenge to milk, soy, and grain was earlier than that of other solid foods. CONCLUSIONS: Our data suggest that our FPIES OFC protocol is safe. Early administration of IVF may prevent the development of hypotension. It is difficult to stratify the risk of severe or delayed reaction based on patient characteristics, and more data are needed to identify those appropriate for home introduction.

\section{Ann Allergy Asthma Immunol. 2019;122(3):314-317.}

\section{Antibiotic choice and methicillin-resistant Staphylococcus aureus rate in children hospitalized for atopic dermatitis.}

\author{
Wang V, Keefer M, Ong PY.
}

BACKGROUND: Systemic antibiotics are commonly used in hospitalized patients with severe atopic dermatitis (AD) exacerbation. However, the antibiotic prescribing patterns are unclear. OBJECTIVE: To compare the prescribing patterns of antibiotics for children who were hospitalized for $\mathrm{AD}$ exacerbation and infectious complications. METHODS: Electronic medical records were reviewed for patients younger than 18 years who were hospitalized for $\mathrm{AD}$ exacerbation or infectious complications based on International Classification of Diseases, Ninth Revision, Clinical Modification and International Classification of Diseases, Tenth Revision, Clinical Modification codes from 2003 to 2018. The following information was obtained: history, physical examination findings, physician discharge summary, antibiotic treatments, serum tests, and wound cultures. The $t$ test was used to compare clinical and laboratory features. RESULTS: A total of 174 patients with AD were included. Seventy patients had AD exacerbation and 104 had infectious complications, including cellulitis, abscesses, invasive infections, and eczema herpeticum. The differences between these 2 groups of patients were further verified by length of stay, serum total $\mathrm{IgE}$ level, and inflammatory markers (C-reactive protein and erythrocyte sedimentation rate). A total of 56 of 70 patients $(80 \%)$ with $\mathrm{AD}$ exacerbation were treated with a systemic antibiotic. Clindamycin and vancomycin together accounted for $88 \%$ of antibiotics on admission for both groups, whereas clindamycin and sulfamethoxazole-trimethoprim were prescribed at similar rates for both groups at discharge. Wound culture results showed that the methicillin-resistant Staphylococcus aureus (MRSA) rate was significantly lower in children with AD exacerbation (22\%) vs infectious complications (39\%). CONCLUSION: Children were treated with a high frequency of anti-MRSA antibiotics for inpatient AD exacerbation and infectious complications. However, the rate of MRSA was significantly lower in children with AD exacerbation. Thus, empiric antibiotic choice for infectious complications may not be appropriate for AD exacerbation. 